\title{
Formal ontology, common sense and cognitive science $\dagger$
}

\author{
BARRY SMITH
}

Department of Philosophy and Centre for Cognitive Science, SUNY, Buffalo, NY 14260-1010,USA.email: phismith@ubvms.cc.buffalo.edu

\begin{abstract}
Common sense is on the one band a certain set of processes of natural cognition-of speaking, reasoning, seeing, and so on. On the other hand common sense is a system of beliefs (of folk physics and folk psychology). Over against both of these is the world of common sense, the world of objects to which the processes of natural cognition and the corresponding belief-contents standardly relate. What are the structures of this world and how does its scientific treatment relate to traditional and contemporary metaphysics and formal ontology? Can we embrace a thesis of common-sense realism to the effect that the world of common sense exists uniquely? Or must we adopt instead a position of cultural relativism which would assign distinct worlds of common sense to each group and epoch? The present paper draws on recent work in the fields of naive and qualitative physics, in perceptual and developmental psychology, and in cognitive anthropology, in order to consider in a new light these and related questions and to draw conclusions for the methodology and philosophical foundations of the cognitive sciences.
\end{abstract}

(C) 1995 Academic Press Limited

\section{Introduction}

Much recent work in cognitive science has taken common sense-in the form of naive physics, folk psychology, or real-world models for natural language processing-as a serious object of scientific inquiry. This paper seeks to clarify the philosophical background to such work. It takes as its starting point the totality of cognitive states and processes that are involved in our everyday thinking, speaking and perceiving and in our everyday traffic with the world in overt bodily action. We shall employ the phrase "natural cognition" to refer to this totality. "Natural cognition" is a generalization of "natural language" in one customary employment of this term. It signifies cognition involving no special (artificial) aids or contexts, cognition that is bound to our everyday actions and perceptions.

It is then necessary to distinguish between:

O1 natural cognition as a totality of processes (within which we can distinguish various sub-totalities of language, reasoning, vision, etc.);

$\mathrm{O} 2$ the more or less coherently organized systems of pre-scientific beliefs (of folk physics, folk psychology, etc.), which are extractable from this totality of

†The present paper was prepared as part of the project "Formalontologische Grunglagen der kinstlichen Intelligenzforschung" sponsored by the Swiss National Foundation for Scientific Research (Bern). It is based in part on work carried out at LADSEB-CNR (Padua) in November and December 1993. Thanks go to J. Brandl, E. Byckling, M. Thalos, and G. White. 
cognitive processes and which can be seen as playing a central role in the organization thereof; $\uparrow$

$\mathrm{O} 3$ the world (the system of reference-objects) to which the cognitive activities and beliefs in $\mathrm{O} 2$ primarily relate.

This gives rise to a corresponding division on the theoretical side between:

T1 sophisticated (scientific) theories of the processes in O1 (for example psychological theories of the workings of the human visual system);

T2 sophisticated (scientific) theories of the naive belief-systems in $\mathrm{O} 2 ; \ddagger$

T3 sophisticated (scientific) theories of the objects in O3.

Unfortunately, none of the listed dimensions are entirely unproblematic, and each is the subject of as yet unresolved debates between different philosophical and methodological camps. Thus many cognitive scientists see work on developing theories under T3 as part and parcel of attempts to develop computer simulations of common-sense reasoning and beliefs under $\mathrm{O} 1$ and $\mathrm{O} 2$. This association-which is manifested in the very terminology of "naive physics", "folk psychology", etc.-has led some to suppose that the scientific investigation of the structures of the common-sense world ought properly to involve the use of less scphisticated logical or mathematical tools than are available to those engaged in scientific investigations of other sorts. $\$$ This assumption is here rejected.

In regard to $\mathrm{O} 1$ there are those who deny that there is such a thing as "natural cognition" at all, holding instead that there are as many different forms of cognitive activity as there are different individuals or cultures, the latter being seen as manifesting ephemeral and non-law-governed variation in all directions. (This might correspond, in the linguistic sphere, to the denial of the idea that "natural language" designates some single, coherent object of scientific investigation, or more specifically to a denial of the thesis that there are universals of natural language.) In regard to $\mathrm{O} 2$ there are philosophers especially in the Wittgenstein camp who have denied that there is anything system- or theory-like in the fabric of beliefs that are manifested in our everyday cognitive behaviour. Rather, Wittgenstein seems to hold, such beliefs are marked by a dependence on context that is in principle unlimitedly nuanced. In regard to $\mathrm{O} 3$, most importantly for our purposes, it has been denied by some that there is a single "world" to which natural cognition can properly be seen as relating. Cultural diversity, on the view in question, has ontological implications to the effect that the members of different cultures live in different worlds. We shall

\footnotetext{
t The relation between $\mathrm{O} 1$ and $\mathrm{O} 2$ is analogous, perhaps, to that betweer parole and langue in Saussurian linguistics.

$\ddagger$ The construction of theories under $\mathrm{T} 2$ may involve a systematic effort at constructing theories out of the belief-systems under 02 . Such belief-systems may thereby be to some degree subjected to improvements of various sorts. What is sometimes referred to as the philosophia perennis (a sort of enduring common core) of Aristotelian and scholastic philosophy is on Sellars' view a "refinement"-in this sense of what Sellars calls the "manifest image"-of common sense (Sellars, 1963: p. 8).

$\S$ See Smith (1994) for a more detailed discussion of this phenomenon. The association has arisen in virtue of the fact that there are two hearts-of simulation, and of theory-which beat within the breast of artificial intelligence research.
} 
spend much of our time in what follows in setting out an alternative to the view that there is a diversity of ontologies generated by the diversity of human cognitive systems. Note that we are interested exclusively in what we shall persist in calling natural cognitive systems. There is no doubt that the specialist cognitive activities of mathematicians, ufologists, Meinongian philosophers and the like, relate to ontologically peculiar worlds. These activities are not, however-in a sense still to be made clear-natural (and mathematicians do not form a natural culture in the sense here at issue).

In fact we shall argue for two somewhat radical theses: 1. Uniqueness: that what we shall call the common-sense world is, modulo certain trivial differences of emphasis and calibration, culturally invariant; and 2. autonomy: that this commonsense world exists independently of human cognitive activities.

Ad 1. It is difficult to state the thesis of cultural invariance in a coherent fashion. Broadly, however, our position can be defined as one of ontological commensurability: two conceptual systems are commensurable in the ontological sense if (a) both can be conceived as systems of delineations of a single objective world, the world in which we live and move, (b) and this world is such as to exist independently of our conceptualizations and delineations. (We shall assume, that this is roughly the same world as is investigated by physics.) Clearly ontological commensurability as thus defined is compatible with skew classifications of a single subject-matter. Ontological communsurability may be more trivial a requirement than other sorts of commensurability. Thus Lakoff (1987: p. 322) lists five different ways in which two conceptual systems can be commensurable: intertranslatability, understandability (one person can understand both alternatives), common use (the same concepts are used in the same ways), framing (situations are "framed" in the same way and there is a frame-by-frame correspondence between the two systems), and organization (the same concepts organized in the same way occur in both systems). Much incommensurability in any of these five senses may be compatible with ontological commensurability.

Ad 2. We shall claim, indeed, that the dependence flows in the direction opposite to that which is favoured by proponents of cultural diversity views, and that human cognitive activities are as they are in large part in virtue of an independently existing environment of medium-sized manipulable objects (scenes, perceptible qualities, etc.) within which these activities are lodged. We thus defend the Gibsonian idea to the effect that work on natural cognition ought properly to go hand in hand with work on the structures of that common-sense world to which, if we are right, natural cognition relates. $\dagger$

This idea is pursued, however, not from the perspective of psychology, as in Gibson's case. Rather we shall seek to formulate the idea by using tools which have been developed by philosophers in recent years working in the field of ontology. It will then turn out that the features of the common-sense world that are culturally invariant on our account can be divided into two groups, of formal-ontological and material-ontological or qualitative features, respectively. We shall discover that both sets of features can best be understood in terms of concepts drawn from the two 
central ontological disciplines of mereology-the theory of part and whole-and topology - the theory of contact, separation and connectedness. $t$

\section{Common-sense realism}

The thesis that there is only one world towards which natural cognition relates is a central plank of what philosophers in the course of history have identified as the doctrine of common-sense realism. This is a doctrine according to which:

(a) we enjoy in our everyday cognitive activities a direct and wide-ranging relational contact with a certain stable region of reality called the common-sense world;

(b) our everyday cognitive activities rest upon a certain core of interconnected beliefs called "common sense" which is in large part true to the common-sense world as it actually is, not least in virtue of the fact that such beliefs and our associated cognitive capacities have arisen through interaction with this world;

(c) this common-sense world exists autonomously, which is to say independently of our cognitive relations to it. Indeed from the perspective of common-sense realism the common-sense world exists entirely independently of human beings. Partial evidence for this thesis is provided by the fact that palaeontology and related disciplines describe this world as it was before human beings existed. Of course this world would lack theoretical interest in a universe populated exclusively by creatures with cognitive capacities radically different from those of human beings. But what these disciplines describe is, nonetheless, such as to exist independently. As Hilbert (1987: p. 19) puts the point in his defence of a realist ontology of colours:

All that is necessary for the objectivity of a property is that objects have or fail to have that property independently of their interactions with perceiving subjects. Color is objective in so far as the colors of objects do not depend on how they appear to an observer or even whether or not there are any observers.

Common-sense realism is a special brand of realism in general, or in other words of the view that the world exists independently of our cognitive relations to it. Realisms differ in their accounts of what the world is that thus exists independently. They are opposed to idealisms of various sorts, for example to linguistic idealism, a view to the effect that the world exists (or has the structure which it has) in virtue of the language we use to speak about it. Subjective idealists hold similarly that the world exists in virtue of our mental activity. Note that all idealists-to the extent that they embrace a position that is capable of being coherently formulated-are in fact also realists of a certain stripe, since all idealists hold that there is something (be it mind, language, or Absolute Spirit) which enjoys autonomous existence.

\section{Appearance and reality}

Our natural cognitive experiences are of course in many cases non-veridical, and thus the common-sense realist must confront the fact of error.

At the same time, however, it must be pointed out that common sense is itself

t These may be extended by an account of orientation, directionality of motion, etc. The materialontological features of the common-sense world are discussed in greater detail in Petitot and Smith (forthcoming), which complements the present paper. 
aware of the many sorts and species of error that are involved in our everyday cognitive endeavours. Thus common sense is not, in spite of its reputation, naive; it draws a systematic distinction between reality and appearance, or in other words between the way the world is and the way the world seems or appears via one or other of the sensory modalities and from the perspective of one or other perceiving subject in one or other context. The thesis that there is only one world towards which natural cognition relates must thus be understood as being compatible with the thesis that there are many different ways in which the world can appear to human subjects in different sorts of circumstances. $t$

Our capacity to sustain the appearance-reality distinction in robust fashion rests on the fact that the common-sense world (O3) is marked by a stability and internal coherence that is higher than that which natural cognition (O1 and $\mathrm{O} 2$ ) itself possesses. This means, first of all, that while the experiences of hearing and touching are vastly different from those of seeing and smelling, the world of what we hear and touch is nonetheless identical with the world of what we see and smell. It means that, in virtue of what psychologists have dubbed the phenomena of constancy (with respect to colour, angle, distance, illumination, etc.), we have the capacity spontaneously to override the perspectival features which pervade our experience. Thus we have no difficulty in grasping the enduringly identical colours, shapes and sizes of material bodies even under quite radical changes in lighting conditions and under quite radical changes in perspective and distance. (Colour is for this reason counted by common sense as no less subject-independent than shape and other primary qualities. $) \div$ Material bodies themselves are similarly grasped spontaneously as retaining their identities even when quite radically deformed or occluded. Certainly there are circumstances in which things go wrong and we misgrasp identity. There exists a repertoire of possibilities in this respect, too, and common sense can at least in many cases recognize and call forth an appropriate ploy for reacting to them, though such ploys need not in every case involve any predetermined recipe for determining the nature of the objects by which one is confronted in given non-standard situations-this much we have learned from discussions of the so-called "frame problem" in work on artificial intelligence.

\section{Perception as discrimination}

The common-sense realist holds that perception is a source of veridical information about the common-sense world. As Neisser (1987; p. 11) puts it: "Under normal circumstances, perception of the local environment is immediate, effortless, and veridical". The putative information supplied by perception is always partial, and sometimes erroneous, but it can in every case be supplemented and corrected by the gathering of further information about the sides of objects we cannot see, about the future behaviour of objects, and so on.

† As Forguson (1989: p. 157) points out, there are four cognitive abilities which are essential to the common-sense view of reality: "... the ability to engage in metarepresentations [representations of our own and others' representations], the ability to make an appearance-reality distinction, the ability to recognize representational diversity, and the ability to recognize representational change; none of these abilities is present in us before 18 months, and the latter three do not appear until about age 4".

$\ddagger$ Forguson (1989). 
It is a further presupposition of the broadly Gibsonian common-sense realism here defended that our everyday unaided perception involves no conceptual or theoretical intermediaries and is in this sense "direct". Perception yields a faithful image of reality in a way which, for Gibson, involves no computation of any kind. It might be argued against this that what counts as an entity for the purposes of perception may depend on what concepts (sortals) are brought to bear in our perceptual experience:

We might not describe a person as having seen, for example, an X-ray tube, unless he possessed an understanding of what an X-ray tube is; but this does not mean that he cannot actually perceive the thing we know to be an X-ray tube, cannot pick it out from its background. The focus of attention does not create entities where none in fact are. We attend selectively to certain features that are there in fact, and they determine what it is possible to perceive. But the act of attention need not depend on a prior conceptual understanding of the things attended to. In the normal case, there are patterns in the stimulus array that are specific to the real entitics around us, and allow us to discriminate them directly. (Kelley, 1986: p. 167)

Perception relates, then, not to the array of qualities as such (in this it is distinguished from sensation), nor to a conceptually or theoretically organized world, but rather to the boundaries, the qualitative gradients, in those sides or portions of the world towards which our perceptual organs are at any given stage directed and to which they are in a certain sense attuned.

Perception is a matter of picking out certain discriminated entities and setting them into relief against their background in such a way as to give us an awareness of entities "as units of existence, identity, and change". (Kelley, 1986: p. 173) Different persons on different occasions have different capacities to discriminate in this regard, and thus our perceptions are subject to differences of granularity, reflecting different levels of organization on the side of the objects themselves. $t$ What, at first, appears to me in a Seurat painting as a green surface will on closer inspection appear as an array of blue and yellow dots. Differences of this sort may imply that the common-sense world that is discriminated by natural cognition may differ from culture to culture (as Eskimos are said--correctly or incorrectly-to have no single word which would correspond to the English word for snow). Even if such variations can be shown to exist, however, they constitute no challenge to our two theses of uniqueness and autonomy of the common-sense world, since they reflect no ontological incompatibility on the side of the objects, but rather differences of granularity or of calibration in our articulation of one and the same world (as one can measure, alternately, in metric and imperial units). The thesis of the autonomy of common-sensical reality may for this reason be compatible even with the idea that frogs might have a common-sense world of their own. The laws governing this world would be different from those governing the world of human common-sense. But the two sets of laws would still be consistent with each other, in the sense that they would reflect cuts through the same reality at different angles or of different calibrations (as one can slice a cheese in different ways).

$\dagger$ Still finer levels of discrimination can be effected via the use of instrumental aids such as microscopes. The objects thereby revealed, however, lie outside the "natural" realm of common sense as here conceived. 


\section{Common sense and physics}

The common-sense realist must also confront the question of the relation between the common-sense world and the world that is described in the textbooks of standard physics. Here again a number of different philosophical alternatives have been mapped out in the course of philosophical time, including the view that it is the common-sense world that is truly autonomous while the world of physics is to be awarded the status of a cultural artifact. $\dagger$ Here, in contrast, we shall assume a thesis to the effect that the common-sense world overlaps substantially with physical reality in the more standard sense. More precisely, we shall assume:

(1) That at least the solid and non-solid stuffs of the common-sense world are properly included within standard physical reality. Common-sense realism is thus of a piece with physical realism understood as the view that physics is a true account of reality at some deep level of structure, and the world of common-sense is capable of being investigated in large part also by standard physical means.

(2) But that there are certain features of the common-sense world of external reality which fall outside the purview of standard physics. Thus while the common-sense world must be compatible with standard physics, it may go beyond physics in certain harmless but important ways. Examples of features peculiar to common sense reality in this sense will be:

(a) formal-ontological structures and relations of certain sorts (for example relations of existential dependence), regions of space and stretches of time, shapes, holes, patterns and other similar structures, natural kinds or types and systems of boundaries, $\neq$

(b) material-ontological features belonging to the qualitative world of colours, tones, etc., conceived as qualities of external things.

(a) and (b) are interrelated, in virtue of the fact that the boundaries salient in human experience are in no small part cases of qualitative discontinuity. $\$$

Note that the above is formulated in relation to the external world of common sense; we leave open the question whether those features of the common-sense world (sense data, feelings, etc.) which fall within the purview of folk psychology are also capable of being described by standard physics.

The thesis that the common-sense world is capable of being investigated in large part by standard physical means may, however, have limited implications. Thus suppose, with Hilbert, that we identify colour with the physical property of surface reflectance. The latter is an objective property and is physically well understood, yet, as Hilbert (1987: p. 120) points out,

it is not reducible to more fundamental physical properties. Fundamentally different physical mechanisms can result in objects that possess very similar dispositions to reffect light. The reflectance of an object is a multiply grounded dispositional property.\|

$\ddagger$ A view of this sort is defended by Husserl in his The Crisis of European Sciences (1970b).

$\ddagger$ All of these entities have some of the qualities of universals: all require individual hosts, vi: their existence is compatible with the existence of an indefinite range of alternative hosts. All of the given entities are in addition in a certain sense too thin to contain matter or energy-they lack divisible bulk and thus they fall outside the purview of the discipline of physics. See Smith (forthcoming).

$\S$ See Petitot and Smith (forthcoming).

\| Note that something similar holds of almost every other standard physical property. 
For this and other reasons many of the sorts of phenomena which are characteristic of the common-sense world are not as such of interest to the physicist. Such phenomena are set into relief in relation to phenomena of other sorts only in virtue of certain largely arbitrary facts concerning the human perceptual system. This does not, however-much confusion amongst idealist philosophers notwithstandingmake them dependent for their existence upon human perceivers.

\section{How could we discover that common sense is false?}

The doctrine of common-sense realism is not merely a doctrine concerning the ontological status and nature of the common-sense world. The doctrine also has an epistemological component, embracing a thesis affirming the existence of a network of actually existing relations between the objects in this world and ourselves as cognizing agents, relations which facilitate veridical cognition: the world and its subjects are as it were in tune with each other. Thus common-sense realism is to be contrasted not only with idealism but also with what might be called asymptotic or utopian realism, a view according to which cognitive access to realia can in principle be achieved, but only in the long run, with the ultimate perfection of our cognitive apparatus, when some future ultimate science will finally match up to the world as it is in itself. Like various forms of idealism, asymptotic realism denies what seems to the common-sense realist to be the evident fact that we are already in direct contact with the world, or with much of the furniture of the world, and have been so for a long time (Moore, 1959: p. 33).

Could common-sense realism be false? One first approach to this question might be to consider what sort of evidence might count against it. Could we have good reasons-perhaps derived from physics-for embracing a thesis to the effect that our common-sense world view is radically, or in its entirety, false? Philosophers and scientists since Galileo have held that we could have such reasons, and there are indeed scientific truths which seem to be well-established and yet to stand in conflict with pre-scientific views of external reality. On the one hand are results such as those obtained by Bozzi which point to certain systematic errors in our lay understanding of the behaviour of pendula and other simple mechanical devices. $t$ Untutored perceivers, when presented with a series of films of pendulums oscillating at a range of natural and unnatural frequencies, will tend to classify as natural frequencies of oscillation which are significantly slower than the frequencies at which the corresponding real pendulums would in fact oscillate. Untutored perceivers similarly make significant errors when asked to predict how the course of motion of a projectile will continue given an initial motion of specified curvature and velocity, and they tend radically to over-emphasize the gradients of uphill and downhill roads along which they travel.

Such data must, of course, be set against the far greater wealth of data in relation to those respects in which lay physics is unproblematically correct in its understanding and even in its predictions of physical behaviour (for example in its prediction that heavy objects will fall downwards when dropped). The investigation of the latter has for obvious reasons been less attractive to psychologists, since it is to a large degree (precisely) a matter of trivial truths accepted by all.

† See Pittenger and Runeson (1990) and the references provided in Smith and Casati (1994). 
But even the Bozzi-style results have consequences only in regard to our untutored apprehension of certain features of the phenomena in question, above all to metric features, and to features of curvature and shape. As concerns mereological, topological and vectorial features (including features relating to orientation and convexity/concavity of motion-paths, etc.), as well as certain qualitative features of the given phenomena, our grasp thereof is in every case-modulo the degree of discrimination which our perceptual organs are capable of achieving-veridical. Yet it will turn out that it is mereology and topology-matters of what is part of what, of what is discontinuous from or moving in relation to what-which serve to delineate and determine the realm of common-sense reality as here understood. The Bozzi-type results must therefore strictly speaking be taken as supporting the claim that common-sense physics is, in the formal- and material-ontological respects which here concern us, in harmony with its object.

More pressing are the seeming incompatibilities between, say, the view of reality that is dictated by quantum mechanics and the view of reality of the layman. It would lead us too far from our main concerns to address this issue in detail here. Suffice it to point out that physicists themselves have well-recognized techniques for explaining the mesoscopic properties of material bodies on the basis of their understanding of the corresponding microphysical structures. (Thus for example there is a well-established physics of colour.t) Indeed, the absence of such techniques or of the means for understanding how the microscopic and mesoscopic worlds are related together would rightly be held to count against the claims of physics to constitute a science of reality. To this extent, then, a correct understanding of physical science requires that it should vindicate the judgments of common sense to a substantial degree.

In this connection it is worth bearing in mind further that, as Sellars points out, standard physical science and the hard sciences in general have their origins in common sense. Thus as Sellars expresses it, "the scientific image cannot replace the manifest image without rejecting its own foundations" (Sellars, 1963: p. 21). The fact that our concepts of, say, elementary particles are able to play a role in facilitating scientific understanding turns at least in part on the fact that such concepts are extrapolations of commonsensical concepts (of relative smallness of size, spherical shape, presence or absence of proper parts, etc.) with which the scientist is familiar from the start. The physicist must in addition appeal to objects and processes of the common-sense world (dials, levers, test-tubes) when testing his theoretical constructions against experience, and in this respect, too, there would seem to be some dependence of the results of the physicist's investigations upon a presupposition of the truth of (much of) common sense.

\section{A note on model-theoretic semantics}

An adequate theory of natural cognition presupposes a theoretical understanding also of the structures of that common-sense world to which nutural cognition relates. This thesis is at odds with the thesis taken for granted by modern philosophersa thesis rarely explicitly formulated-to the effect that the tasks of semantics and 
theory of reference are most properly to be realized by investigating not the concrete world of common sense, but certain sorts of abstract structures. This applies very clearly, for example, to those formal philosophers working in the tradition of Montague, but the mentioned thesis is much more widely held, and it is nowadays almost always taken for granted that the job of the semantic theorist involves the construction of special set-theoretic models. The latter serve as surrogates for the things and events of the common-sense world, so that semantic investigation of the sentences of natural languages (and of the thoughts which these sentences express) remains at one remove from the world towards which on a realist understanding these sentences relate. This is a reasonable outcome if the aim of semantics is one of characterizing linguistic structure in its own right, i.e. independently of its concrete referential application. It is also reasonable if the aim of semantics is one of specifying the meanings of those syncategorematic terms-for example quantifiers and other logical constants - which play a role equally in all domains of discourse. It is through a treatment of such functional meanings-and this is the glory of post-Tarskian semantics - that set-theoretic models are able to help us to understand the truth-behaviour of the logically simple sentences from out of which they are constructed. Where, however, our concern is precisely with the world-embrangled meanings of a natural language and with the associated world-embrangled systems of natural cognition, then this sort of semantic investigation must at the very least be supplemented by inquiries of a different sort, inquiries pertaining precisely to the structures of the common-sense world. Only thus, indeed, will we be in a position to establish the adequacy of set-theoretic models of natural language of the more usual sort.

\section{Against methodological solipsism}

The thesis that semantics ought properly to proceed by dealing only with certain abstract models tailored to and dependent upon the structures of the languages with which the semantic theorist deals, has its analogue, in the psychological sphere, in the doctrine of "representationalism" or "internalism". This doctrine, which lies at the heart of much contemporary cognitive science, has roots in the view of Descartes to the effect that the external world could be in every respect completely different from what it is (could even not exist) and yet our thoughts would remain exactly the same. Perhaps the strongest statement of the internalist thesis has been formulated by Fodor (1980) in his paper on the methodology of cognitive psychology. If, Fodor argues, our psychological processes were to be conceived as relational in structure in the sense that they are intrinsically of or about certain corresponding real-world objects, then the investigation of such processes would have to involve the investigation also of those objectual targets themselves. This, however, would rule out the possibility of a science of psychology. For the latter, before it could formulate laws of its own, would need to presuppose a theory of the objects of thought, and this, as Fodor puts it, would have to be a theory of everything, a universal pan-science embracing all other sciences as constituent disciplines. A nomological psychology would have to "attempt to specify environmental objects in a vocabulary such that environment/organism relations are law-instantiating when so described". But "we have no access to such a vocabulary prior to the elaboration 
(completion?) of the non-psychological sciences" (Fodor, 1980: p. 300). Thus we could not construct a naturalistic psychology of reference unless we had some way of saying for example what salt is, which of its properties determine its causal relations with other things and with ourselves, and so on ad indefinitum.

Fodor's argument, if it is valid, poses a threat to the common-sense realist's thesis that cognitive experience is relational in structure, for the latter implies that cognitive processes do indeed reach out to the concrete things and processes of the common-sense world and that an adequate science of cognition, if such is possible, would have to be in a position to do justice to just this fact. Fodor's argument can be countered, however, if common-sense realism is indeed correct. For the argument presupposes the absence, at every non-ultimate stage in the development of science, of any scientifically well-established account of the real-world objects which serve as the objects of our thoughts. It presupposes, that is to say, what we have called "asymptotic realism" above. The common-sense realist, on the other hand, insists that we are already, in the great mass of our everyday cognitive experiences, in unproblematic and systematic cognitive contact with a certain stable domain of objects in the world called "common-sense reality". On this basis it becomes possible to challenge the idea that a science of the objects of thought must be the whole of science, as Fodor presupposes. For to do justice, scientifically, to the objects of natural cognition it would be sufficient to develop merely that small portion of the science of everything which relates to common-sense objects. It is possible, after all, to understand the psychology of reference to salt without knowing anything about the chemical properties of salt, by appealing only to features like: stuff which comes in grains, white, sour tasting, soluble, etc. It is not part of the business of the science of natural cognition to concern itself with the chemical and other sorts of specifications of those commonsensical objects of thought with which it is concerned. Indeed if, as we have suggested, the stuffs and qualities of the common-sense world are a part of the world of standard physics, then it will follow as a matter of course that common-sense experience can reveal only a certain portion or side or aspect of the common-sense world as strictly understood. $\dagger$

A response to Fodor's (1980) argument along these lines is not without its price. It will involve rejecting the goal of a nomological science of natural cognition. The physical universe is causally closed; it is such as to constitute a single, harmonious, organized totality, the events and processes constituting it being in principle explicable in terms of prior events and processes and corresponding laws. Our assumption concerning the relations between the common-sense world and the world of standard physics implies, too, that all events and processes of the common-sense world are caused. But it does not imply that all such events and processes are caused by events and processes within the common-sense world itself, and nor a fortiori, does it imply that they all have causal explanations formulable in terms relating only to common-sense experience. The common-sense world is accordingly not causally closed. Our mental experiences, above all, form part of the

† Thus for example: "Perception does not reveal the whole truth about colors and the truth it does reveal is delimited by the characteristics of our perceptual systems. Human perception of all properties, not just colors, is indeterminate in the sense that it only delivers partial information about the fully determinate qualities that objects possess" (Hilbert, 1987: p. 27). 
common-sense world; not, however, the neurophysiological events and processes which play a role in causing such experiences. $f$

The common-sense world is moreover a world of salient structures in the sense of Gibson (1979) or Thom (1988), and what is salient for an organism in an environment is not capable of being grasped nomologically (a pound note and an equivalent number of coins have the same cognitive significance, yet share no physical or chemical properties in common). Fodor and Pylyshyn (1981) are very clear on this point in their critique of ecological psychology in the sense of Gibson. The information in the environment that is salient for the subject is not capable of being described in physical terms. The symbolic mental representations which appear at the central cognitive systems are the result of processes which cannot be understood as taking place in accordance with strict laws.

On the other hand, however, it is far from clear that even those research programmes in psychology which have been pursued on the basis of methodological solipsism have yielded results which lend substance to the idea that a nomological science of psychology is indeed capable of being developed. In its most fully worked out versions, psychology appears, rather, as a descriptive, taxonomical science, approximating more closely to (say) linguistics than to physics or chemistry. Predictions of a certain sort are possible within the science of natural cognition, but the predictions in question are of the types of structures realized and of the types of possible development, rather than of any quantitatively precisely specific outcomes of the sort that are possible in at least certain parts of physics.

There is, then, room for a naturalistic science of psychology that would take account also of the objects of everyday cognition, a science that would fall between the two extremes of Cartesian psychology on the one hand and the universalistic pan-science described by Fodor on the other. It can indeed be argued that only the pursuit of this middle ground can yield any sort of understanding of cognitive phenomena in theoretical terms. For as Kelley (1986) points out:

we discover which aspects of what we perceive have to be assigned to the sensory system only by identifying in the object the intrinsic features to which the senses are responding. Thus at every stage of the investigation, knowledge of how the senses function presupposes knowledge about the objects they respond to. $\$$

\section{Folk psychology and folk ontology}

The set of common-sense beliefs about external reality is of course part of a wider totality which includes also common-sense linguistics, common-sense economics, a common-sense theory of ethics and law and table-manners as well as much else, and it is by no means easily detachable from this wider background. Really existing common sense is a jumble of many different things, ranging from transient and culture-dependent prejudices to universally accepted truths as trivial as: an A is an

\footnotetext{
† Common-sense "does not attempt to explain how beliefs and desires can have the causal powers we attribute to them". Moreover, it is "silent about the detailed microstructure of the mind-independent world which it posits as being thought about and perceptually experienced by sentient beings" (Forguson, 1989: p. 24).

$\ddagger$ See Petitot and Smith (1990, forthcoming).

$\$$ Kelley (1986: p. 43), referring to the realism expounded in Duncker (1947).
} 
A. Common-sense includes a massive storehouse of factual knowledge about colours and sounds, about time and space, about what foods are edible and what animals are dangerous. How, then, can we bring some necessary order into this jumble?

Recall, first of all, that in developing our theory of the common-sense world we shall not confine ourselves to the resources available at the level of common sense itself. Rather, we shall use in our theorizing the most sophisticated instruments available, paying attention to common-sense beliefs and reasoning processes only insofar as these help us to determine the nature and limits of that common-sense reality which is the proper object of our investigations.

The common-sense realist holds that it is possible to develop a theory of the common-sense world that is true. (It was investigations of this subject-matter which predominated among metaphysicians and natural philosophers in the time before Galileo.) A parallel intuition is defended by those who give credence to the idea that our everyday psychological beliefs, too, constitute a bona fide systern of truths. Indeed each of the physical issues dealt with here seems to have an equivalent in the issues addressed by philosophers and others under the heading of "folk psychology". + There, too, one finds a wide variety of true and false beliefs that have been maintained by human beings at different times and places. The existence of false beliefs about the mind does not of itself, however, imply that it is impossible to disengage therefrom some core of true folk-psychological beliefs which might further claim a truly scientific status when suitably formulated.

Similarly, I shall claim, the existence of more or less folksy belief-sets pertaining to the external world of human perception and action is not of itself a sufficient warrant for rejecting the idea of a (true) theory of this world. Moreover if, as some hold, it is a worthy project to attempt to prune away the more folksy bits of common-sense psychology in order thereby to establish, as far as is possible, the laws governing the mental domain of beliefs and desires, then it ought, surely, to be a no less worthy project to attempt to prune away the more folksy bits of common-sense physics in order to establish the laws governing that domain of commonsensical things and events to which such beliefs and desires primarily relate.

\section{Anthropology}

The first clue to the nature of such pruning is provided by anthropology, and we shall in fact begin our pruning by restricting our attentions, as best we can, exclusively to those beliefs which are common to all cultures and societies. Each culture has of course its own culture-specific common-sense beliefs pertaining to external reality. Anthropologists have, however, established that there is a nontrivial core of such beliefs which is, modulo variations in emphasis and calibration referred to above, common to all societies. Such beliefs belong to what the anthropologist Robin Horton calls "primary" theory, as contrasted with the "secondary" theories of a religious, mythical or scientific nature which pertain to what lies beyond or behind the world that is immediately given in perception and action. As Horton (1982: p. 228) puts it:

† This is clearly brought out by D'Andrade (1987), who incidentally reveals also the remarkable similarity between folk-psychological models and the work of traditional philosophers on the structures of mind. 
Primary theory really does not differ very much from community to community or from culture to culture. A particular version of it may be greatly developed in its coverage of one area of experience, and rather undeveloped in its coverage of another. These differences notwithstanding, however, the overall framework remains the same. In this respect, it provides the cross-cultural voyager with his intellectual bridgehead. Primary theory gives the world a foreground filled with middle-sized (say between a hundred times as large and a hundred times as small as human beings), enduring, solid objects. These objects are interrelated, indeed interdefined, in terms of a "push-pull" conception of causality, in which spatial and temporal contiguity are seen as crucial to the transmission of change. They are related spatially in terms of five dichotomies: "left/right"; "above"/"below", "in-front-of"/"behind"; "inside"/"outside"; "contiguous"/"separate". And temporally in terms of one trichotomy "before"/"at the same time"/"after". Finally, primary theory makes two major distinctions amongst its objects: first, that between human beings and other objects; and second among human beings, that between self and others. In the case of secondary theory, differences of emphasis and degree give place to startling differences in kind as between community and community, culture and culture. For example, the Western anthropologist brought up with a purely mechanistic view of the world may find the spiritualistic world-view of an African community alien in the extreme.

From the anthropological perspective, moreover, we can understand that there are good reasons for the existence of this universal primary theory: the remarkable facility which humans manifest in reasoning and acting on the level of everyday experience can be accounted for precisely by the existence of stable structures on the side of reality to which their thoughts and actions are attuned. The ontology of the common-sense world might thereby play a role in explaining the acquisition, adaptability and success of our common-sense beliefs. Compare, on this, Campbell (1988: p. 171):

Even if our perceptual world is a world of Appearance, rather than Reality, it is not a world of mere Appearance. It is a perceived world whose characteristics are systematically related to the real characteristics of the real world. Otherwise, we would not be here. The survival value of perceptual reliability is so overwhelming that the first creatures to attain it would inherit their niche.

\section{Developmental psychology}

The idea that there is a non-trivial, true theory of reality that is common to all people has a long history, making itself felt already in connection with doctrines on the natural law in Ulpian and Aquinas (Holthoon, 1987). The idea is often formulated in developmental terms, as for example in the work of the French Jesuit Claude Buffier who, in arguing against what he saw as the threat of scepticism initiated by the Cartesian philosophy, presents a view of common sense as a matter of certain dispositions given by nature to all men or, manifestly, to the great majority, so that when they have attained the use of reason they may pass common and uniform judgment concerning various objects of private opinion individually perceived. This judgment is not the consequence of any prior principle. $f$

Or as Thomas Reid would have it: common-sense beliefs, in being shared by all of us past the stage of infancy, are such that they form an inevitatile presupposition to 
our interchange with others. These beliefs are taken so much for granted that whatever is contrary to them appears not false but absurd.

Evidence from developmental psychology for the existence of a common core of common-sense beliefs that is shared by all cultures can indeed by used to supplement anthropological evidence of the sort that is marshalled by Horton (1982). Thus as Forguson shows in detail, very many of the central features of common sense are acquired, en bloc, at around the age of four, when the child manifests also a new quality of behaviour and of interaction with his fellows. And then, Forguson (1989: p. 175) argues, "The truth of our common-sense beliefs is the best explanation of the differential performance of 3-year-olds and adults in experimental conditions and also in natural settings."

A certain capacity to apprehend those basic structures of reality which are relevant to its survival must indeed be inborn in any organism capable of learning. The human capacity to distinguish colours or shapes, for example, or to recognize similarities, or to find some experiences more rewarding than others, could not have been learned, for such capacities are presupposed by any process of learning which would be conceivable for human beings. As Horton (1982: p. 234) points out:

after a long period of flirtation with a tabula rasa model of higher brain centres, human biologists seem inclined by more recent evidence to think that the brain has elements of genetically-programmed structure and physiology particularly fitted to seeing, thinking and talking in primary-theoretical terms. Again, the psycho-linguists, contemplating the extraordinary facility with which children learn primary-theoretical discourse under a minimum of deliberate instruction, have felt compelled to invoke an element of genetic programming to account for this phenomenon.

Thus we possess innately not merely for example the concepts of identity and difference, but also, and no less importantly, the capacity to apply these concepts correctly (in normal cases) to the things and events by which we are confronted in our everyday experience. $\dagger$ We possess innately the belief that there exists a world of objects and events external to and independent of our conscious experience. We possess innately also the belief that this world is a common, public possession, equally accessible to all, and we are born with an innate disposition not merely to employ language but also to take it for a reliable means of mapping the types and individuals of this world. Isolated individuals (adolescents, philosophers, underground men) may fall away from beliefs of this sort for specific reasons of their own; as regards typical and normal human beings in typical and normal cultures and societies, however, the beliefs in question are entirely unproblematic. $\neq$

Of course the fact of innateness constitutes in itself no evidence for the truth of any single belief. We do however begin to have evidence of this sort when we

† This implies also an innate logic, as is shown in detail by Macnamara (1986). And it also implies an innate geometry, as was recognized by Thomas Reid, whose nativist philosophy of geometry has been summarized in terms of three main theses as follows (Daniels, 1989: p. 110f; italics added):

(1) The notions which we have of mathematical points, lines, angles, and figures are determined by our constitutions.

(2) We can see the necessary relations holding between points, lines, angles, and figures because of a natively endowed ability.

(3) We can "form geometrical conclusions, built upon self-evident principles" (Inquiry, p. 297, Stewart, Ed., Works vol. 1)

$\ddagger$ On the notions of typicality and normalcy here see Smith (1995). 
examine the mentioned beliefs themselves on the systemic level. Thus such evidence is provided by the fact (1) that the expressions of common-sense beliefs are so readily translatable from language to language, (2) that judgments expressing such beliefs are marked by a widespread unforced agreement, and (3) that the inputs of the various senses cohere so painlessly. It is the job of empirical linguists and psychologists to determine in detail the precise degree of such agreement. If, however, common sense truly is a common possession of all human beings who have reached a certain stage of maturity, then it will follow that each one of us is as qualified as the other to set forth at least the bare bones of the theory of this reality. We are allowed, as Hayes (1985; pp. 13ff.) points out, to take seriously the results of something like the play of intuition in this regard.

\section{Cultural relativism}

What of arguments to the effect that the common-sense world is a cultural artifact of Western science, a framework for organizing data which is presupposed by Western linguists, anthropologists and others and which is as it were foisted on the data obtained in the investigation of alien cultures in such a way as to make it appear that the common-sense world thereby "discovered" amounted to a cultural invariant? Views of this sort seem to be implied by the work of philosophers such as Quine, whose thesis of the inscrutability of reference might be held to support the claim that we can never know the ontology of an alien interlocutor since we can never enjoy data that is free of our own ontological imputations. As Kelley (1986) argues, however, Quine's very formulation of his own thesis is inconsistent, since it uses terms such as "language", "reference" etc. as if the referents of these terms were themselves scrutable. Linguistic idealism in general is, as Kelley (1986: p. 193) points out,

incompatible with the knowledge used to explain and defend it. Linguistic idealists rely on a theory of human beings as objects of scientific knowledge, including theories of how language is learned and social practices inculcated, to explain in what sense the objects of knowledge and the truth of propositions depend on our conceptual scheme, and to justify their claim that they do so depend

On what basis can such theories of learning, etc. be justified, however, in a way which will not reinstate the entire autonomous and objective world of human beings, their cognitive activities, environment, and so on, which the linguistic idealist claims to be a mere artefact of a certain culture?

The predicament of the linguistic idealist is similar, in fact, to that of Durkheim in his work on suicide. Durkheim sought on the one hand to criticize the commonsense concept of suicide, but he was on the other hand

forced by his whole argument to inadvertently rely on the common-sense meanings of each of his fundamental variables (intention, suicide, education, and so on) for the relations he believed existed between those variables and for his explanations of those relations. (Douglas, 1973: pp. ix-x)

Something similar holds, too, in relation to the apparent testimony of historical investigations which, as Husserl (1970a: p. 373$)$ puts it in his own inimitable fashion:

seem to bear witness to the relativity of everything historical, of all historically 
developed world-apperceptions, right back to those of the "primitive" tribes. Every people, large or small, has its world in which, for that people, everything fits well together, whether in mythical-magical or in European-rational terms, and in which everything can be explained perfectly.

For consider what is involved in establishing just those historical facts which are held to support the relativistic point of view:

\begin{abstract}
Does not the undertaking of a humanistic science of "how it really was" contain a presupposition taken for granted, a validity-ground never observed, never made thematic, of a strictly unassailable self-evidence, without which historical inquiry would be a meaningless enterprise? All questioning and demonstrating which is in the usual sense historical presupposes history as the universal horizon of questioning, not explicitly, but still as a horizon of implicit certainty, which, in spite of all vague backgroundindeterminacy, is the presupposition of all determinability, or of all intention to seek and to establish determined facts. (Husserl, 1970a, p. 373)
\end{abstract}

Primary theory provides, in Horton's (1982) terms, a bridgehead for the investigation of alien cultures. It provides a bridgehead also, as we now see, for historical investigations of our own culture and also for archaeological and palaeontological investigations of the prehistoric world. The theory of the common-sense world can be seen as being extended in different ways by each of these disciplines, all of which relate to objects on the same mesoscopic level. The common-sense world contains a rich variety of different sorts of causal aggregation, of integral wholes and of organic unities of different sorts. $\dagger$ The point where the theory of the common-sense world begins to treat in systematic ways of these causal aggregations and assemblies is a point of transition into other more specialized disciplines (of geography, geology, anatomy, engineering, and so on) which can also be seen as being extensions of the theory of the common-sense world. Legal theory, too (a theory of people, actions, property) has a similar status, and the same can be said also of many other social sciences.

\title{
13. Towards a theory of the common-sense world
}

Primary theory, to repeat, is a matter of beliefs relating to the objects of direct perceptions. This means: (1) perceptions which do not involve the interpolation of any theory or interpretation, perceptions which are integrated directly (physiologically), rather than via some conceptually mediated process of deduction or inference; (2) perceptions which are typical or generic, in the sense that they do not involve special instruments or apparatus or special circumstances-as contrasted e.g. with perceptual experiences in the cinema or in the psychology laboratory or under special chemical influence. Such special cases are not significant from the point of view of the specification and delineation of the common-sense world (a state of affairs parallel, in some ways, to that which obtains in the field of research into linguistic universals). For the common-sense world is delineated by our beliefs about what happens in mesoscopic reality in most cases and most of the time. It is oriented, in oiher words, about the focal instances of the phenomena of the everyday world, rather than about non-standard or deviant phenomena. 
How, now, to begin developing such a theory of the reality that is given in direct perception, including a theory of what is standard, generic, normal, etc.? Such a theory must be at least consistent with physics proper, and physical considerations may in this way affect what is admitted as standard/generic, etc., and in this way also be relevant to the process of specification and delineation of the common-sense world. Thus our determination of what is to count as a generic experience may depend to some extent on our understanding of the relevant physical and physiological facts. All of this implies that the task of delineation of the common-sense world is not to be approached in a Cartesian or aprioristic spirit (for example in the way that this was attempted by Husserl (1970b) in his Crisis). That an experience is generic is not a property of the experience itself which we can read off as it were phenomenologically from within. The qualities of genericity and directness are rather in the end capable of being fixed only outside naive experience, via a sophisticated theory involving also the appeal to externalistic data. The theory of the common-sense world (like the theory of natural cognition in general) is accordingly an empirical theory, and even the process of specifying the borders of this world need not be capable of being completed in a single step. Rather, we can expect to face a certain amount of tacking back and forth. The ontological side of the theory may have to be corrected and given more concrete shape on the basis of psychological considerations relating to relevant commonsensical beliefs. Our theoretical account of these beliefs themselves will however be influenced by physical considerations and by the degree of our success in providing an ontological theory of the corresponding objects, so that we shall have to move in two directions alternately (from $\mathrm{O} 1 / \mathrm{O} 2$ to $\mathrm{O} 3$ and back again). The success of our efforts at mutual adjustment can then be gauged only by examining the degree to which they yield a good total picture of common-sense reality which is at one and the same time theoretically coherent (robust and fruitful in its applications) and faithful to our starting-point in natural cognition. This will in turn provide evidence for the general standpoint of common-sense realism as this has been defended in the foregoing.

\section{Mereology}

The common-sense world is neither a world consisting exclusively of atoms or elements in the sense of Leibniz or Mach, not is it a single, unitary whole in the sense of Spinoza or Bradley. Rather, it is a world which is characterized by articulations or segmentations at different levels. The most prominent organizing principles of the common-sense world are thus the relations of mereology $\longrightarrow$ of part to whole, or part to part within a single whole, of identity, overlapping and discreteness. There are two traditions in the logical and philosophical investigation of such relations. On the one hand there is what might be called the Lesniewski tradition, after the Polish logician Stanislaw Lesniewski, who produced the first formal axiomatic theory of part-whole relations in 1916. On the other hand is what might be called the Brentano tradition, after the 19th-century psychologist and metaphysician Franz Brentano (1976), whose thinking influenced not only Edmund Husserl, the founder of phenomenology but also the work on types of unity and organization by Gestalt psychologists and others in the early decades of this century.

The two traditions are not entirely separate. Lesniewski was himself influenced 
by Husserl's masterpiece, the Logical Investigations of $1900 / 01, \dagger$ and the ideas on the formal ontology of part and whole developed by Husserl in this work are influenced, like those of Lesniewski, by the work of Boole, Schröder, Bolzano and other 19th century mathematicians working in the field of the algebra of logic.

Work in the Lesniewski tradition leans philosophically in the direction of nominalism $\ddagger$ and extensionalism. $\S$ This tradition includes the ideas on part and whole of Leonard, Goodman, Quine and Joseph Woodger, and also the work of latter-day proponents of "theories of aggregates" designed to supplement what is effectively the Lesniewski theory by appealing to some of the power of the theory of sets. \|

The Brentano tradition is more willing than its Lesniewskian mereological counterpart to admit entities of different ontological sorts or categories, and it is in other respects, too, a richer and more inclusive theory. Thus the Lesniewski tradition constructs its mereology on the basis of a single primitive relation of part to whole. This relation generates a theory which proves to be very simple from the mathematical point of view: it involves no more complexity than is to be found in a Boolean algebra.

The Brentano tradition, on the other hand, deals not only with ("vertical") relations between parts and their wholes, but also with the ("lateral") relations among the parts of a single whole. To put the matter simply and crudely: some parts of a whole exist merely side by side, can be destroyed, ot removed from the whole, without detriment to the residue. A whole all of whose parts manifest exclusively such side-by-sidedness relations with each other is called a heap or aggregate or, more technically, a purely summative whole. In many wholes, however, and in all wholes manifesting any kind of unity, certain parts stand to each other in relations of what Husserl calls necessary dependence (which is sometimes, but not always, necessary interdependence). Such parts cannot, as a matter of necessity, exist, except in association with their complementary parts in a whole of the given type. There is a wide variety of such lateral dependence relations, giving rise to an equally large variety of different types of whole which the Lesniewski approach is unable to distinguish. The theory of lateral, part-part relations can however be combined smoothly with a vertical mereological theory of the Lesniewski type in such a way as to produce a radical increase in descriptive power. The theory is still limited, however. Thus it is impossible, within the mereological framework, to deal with numbers or with finite trees and with recursively nested finite structures in general. Set theory will, then, serve us better with respect to structures such as this. On the other hand, however, it is necessary to insist that mereology has advantages of its

† It should be pointed out, though, that Lesniewski was influenced by those sections of this work which deal with applications of Husserl's theory of part and whole to the domain of grammatical categories.

$\ddagger$ Two overlapping senses of nominalism can be distinguished. On the one hand is the view according to which everything that exists is an individual spatio-temporal object. It is contrasted with views according to which there exist also universals, types and other abstract entities. On the other hand is the view that universals are linguistic entities.

\&xtensionalism, for present purposes, amounts to the view that the identity of a whole is determined exclusively by the identity of its parts (and thus independently of any considerations of the way in which the whole is structured or organized). If we write " $x \leq y$ " for " $x$ is a part of $y$ " then extensionalism can be expressed formally by means of an axiom to the effect that $\forall x \forall y(\forall z[z \leq x \equiv z \leq y] \equiv x=y)$.

II See for example, Bunt (1979). For a general survey see Simons (1987). 
own as an instrument of ontology. For the application of set theory to a subject-matter presupposes the isolation of some basic level of Urelemente in such a way as to make possible simulation of the structures appearing on higher levels by means of sets of successively higher types. If, however, as holds in the case of investigations of the ontology of the common-sense world, we are dealing with mesoscopic entities and with their mesoscopic constituents (the latter the products of more or less arbitrary real or imagined division along a variety of clistinct axes), then there are no Urelemente to serve as our starting-point, and only something like the mereological approach gives us the possibility of representing the entities in our domain without committing ourselves a priori to an analysis of internal structures in terms of atoms. $\dagger$

Certainly it need not be necessary that the choice of basic objects be unique; the general case may be like picking a basis for a vector space, which is merely a notational convenience, and can be done in innumerably many ways. Thus it is the vector space structure of, for example, forces that has physical significance; our choice of axes might be pragmatically motivated in a given situation, but per se it has no physical significance. So, too, it may be with the choice of Urelemente in a set theory constructed for purposes of ontology. Even on these terms, however, the resulting account will fall short of mereology, which allows us to represent the entities in our domain in a way which captures also (and for free, as it were) those basic mereological relations which are so central to the structure of this domain in virtue of its simultaneous upward and downward complexity: for each thing or process in the common-sense world may not only be involved with other things and processes to form more complex unities of various kinds, but may also manifest parts of its own which may likewise be involved with other things and processes on a lower level.

Sets are moreover abstract entities: they are not localized in space, and they are subject to a criterion of identity which implies that they are in a certain sense outside history, and are certainly not subject to the sorts of historical changes-including beginning and ceasing to exist-which affect their members: even sets of concrete, changing objects are not such as to change in the course of time, so that it is as if they inhabit a variety of set-theoretical heaven. The things and processes of the common-sense world, however, are both concrete and localized (they are objects of perception and bodily manipulation). For this reason, again, therefore, the cognitive theorist who works with set-theoretical models is faced with the problem of explaining how the abstracta he countenances in his theory can be connected up with the concrete things and processes with which they are ultimately associated.

Sets are further non-integrated entities: any objects, whether real or ideal, abstract or concrete, can (all too) easily be unified together set-theoretically, for example

†The step from set theory to genuine ontology may in certain cases be unproblematic. Thus as "Hayes (1985: p. 10, no. 4) points out, a set-theoretic (Tarskian) model of a theory "can 'be a piece of reality": If I have a blocks-world axiomatization which has three block-tokens, "A", "B", and " $C$ " and if I have a (real, physical) table in front of me, with three (real, physical) wooden blocks on it, then the set of those three blocks can be the set of entities of a model of the axjomatization. There is nothing in the model theory of first-order logic which a priori prevents the real world being a model of an axiom system.

The suggestion works, however, only because we have here candidate Urelemente which are all too easily distinguished, and whose internal structure is irtelevant to the theory in hand. Such cases are unusual, and certainly do not suffice for the purposes of a general theory of common-sense reality. 
within the confines of a simple ordered pair. It is perhaps the most unfortunate consequence of the role of set theory as the primary (and sometimes exclusive) instrument for ontology that the problem of unification (of integral wholes) has been addressed so rarely.

One natural way to approach sets is in terms of a theory of structures capable of being regarded as generated by operations of certain types. For example, the operation of counting can be regarded as an operation on groups of individuals: such groups of individuals are, precisely, sets, and what we need to be able to talk about sets is to be able to form individuals into collections and to have a notion of equality for elements of those collections. Similarly, the operation of enumeration corresponds to the structure of lists, the operation of counting with multiplicities corresponds to the structure of multisets, the operation of assembling things into a hierarchy corresponds to the structure of trees. Mereology in contrast deals with less complex structures of more mundane varieties. Mereology is a decidable theory, which means that one cannot encode arbitrarily complex structures within its framework. At the more restricted level where mereology can properly operate, there is more diversity than there is higher up; we cannot represent these more basic structures in terms of one another with the same facility that one can represent trees in terms of sets (or vice versa). But this has meant that, historically, set theory has been treated as fundamental in a rather misleading sense; that is, it has been treated as a sort of basic structure which other structures were made of. This is a misconception that has had a pernicious effect, e.g. on the history of mathematics.

The most important lesson to be drawn from all of this from our point of view is the following. Set-theoretic structure provides no basis for an understanding of the many and varied sorts of genuine unification (causal, biological, psychological, artefactual, institutional) by which the common-sense world is structured. It is to the understanding of these distinct modes of unification and of the associated genuine separation that much of the effort in developing an adequate ontological theory of the common-sense world will have to be addressed.

\section{Substance and accident: fragments of the theory}

We conclude by providing, for illustrative purposes, some fragments of a theory of the common-sense world, drawing primarily on sources on the history of philosophy and above all on the work of Aristotle. Even the most cursory perusal of the characteristic structures of natural language, perception and action reveals that the world of common sense is organized centrally around bodies, things or substances. These substances are three-dimensional, they have closed two-dimensional surfaces, a certain stock of qualities (of colour, temperature, hardness, etc.), and they occupy a continuous series of volumes of space. $\dagger$ Substances endure through time. This shows that while the common-sense beliefs that are of interest to us relate primarily to perceptual experience, such experience could not suffice alone as a principle of demarcation of common-sense reality, since already the notion of substance goes beyond what is given immediately to perception. The experience of acting, too, will

† cf. the discussion of "singling out" in Wiggins (1980): singling out for substances means singling out not just a volume of space, but a series of volumes of space. 
play a role in this respect, allied perhaps with certain sorts of imagination. (Thus it is the fact that we can imagine performing certain actions on matter that leads us to the commonsensical belief that matter is continuous.) Imagination seems to be involved also in supporting the belief that objects retain their colours even when unperceived and even in the absence of all illumination.

Substances are involved in processes of motion and change of different sorts and they have specific sorts of causal powers and specific sorts of natural development. Substances are, in Aristotle's terms, "one by a process of nature"'. A substance has the unity of a living thing. Hence it enjoys a certain natural completeness or rounded-offness, both in contrast to parts of things and in contrast to heaps or masses of things. $t$ Substances exist continuously and identically through time, existing in their totality in every moment at which they exist at all. Processes, in contrast, unfold in time, existing in no moment of time in their totality. $\neq$ Processes and qualities belong to the wider category of what Aristotle called accidents, embracing e.g. a redness, a specific taste of cheese here and now on my plate, a fall, a blush, a whistle, a salute, a specific waving of a flag (many accidents are, it will be clear, events in the terminology of contemporary analytic philosophy). There are no punctually existing things, as there are punctual events (for example beginnings, endings, judgings, decidings, and instantaneous changes of other sorts).

Accidents are existentially dependent upon their substances; they are necessarily such that they cannot exist unless the substances exist in which (or in relation to which) they occur. Some events and processes (for instance kisses, fights, conversations, promisings, weddings, etc.) are in addition relational, in the sense that they depend for their existence on a plurality of substances. $\$$ Accidents in general may be classified as either relational or non-relational. Substances thereby serve to individuate the accidents which inhere in them, to make them the entities (this colour, that headache) that they are./ Substances are such that, while remaining numerically one and the same, they can admit of contrary accidents at different times. A substance has no temporal parts: the first 10 years of my life are a part of my life and not a part of me. As our ordinary forms of language confirm, it is events and processes, not things, that have temporal parts. The substances of the common-sense world are in fact distinguished from accidents above all in the fact

$\dagger$ Met. 1040b5-16, 1041b28-31, 1052a22ff., 1070b36-1071a4, Cat., 1b5. See also Met., 1042b15-32, for other kinds of unity. Aristotle held that the paradigmatic (if not the exclusive) examples of substances are living creatures, and particularly animals. (It was a radical step in the history of philosophy to the dualist ontology, according to which there are not only material substances in something like the Aristotelian sense but also mental substances, minds, souls or egos.)

$\$$ Thus Kant: "I find that in all ages, not only the philosopher but even the common understanding (gemeiner Verstand), have recognized this permanence as a substratum of all change of appearances, and will always assume it to be indubitable. The only difference is that the philosopher expresses himself in this matter somewhat more definitely, in that he says: throughout all changes in the world substance remains, and that only the accidents change".

This proposition, Kant goes on, belongs "at the head of those laws of nature which are pure and obtain completely a prioni." (Critique of Pure Reason, A 184, Kemp Smith translation amended slightly.)

$\S$ See Mulligan and Smith (1986: 1.2ff). Relational accidents were recognized as such neither by Aristotle nor by at least some of his successors up to and including Leibniz (and indeed beyond). They were, however, acknowledged by Kant (Critique of Pure Reason, A 211f.)

|l Cat., 2 b1 ff.

I Cat., $4 \mathrm{a} 10$. 
that the latter are made up of or constituted by their successive temporal phases: thus my 10-minute headache can be seen as being made up of two successive 5 -minute headache-stages. I myself however am not made up in this way of, say, two successive phases of my life (where I might perfectly well be seen as being made up of limbs, organs and other substantial bodily parts, as also of cells, of molecules, and so on).

Substances are in virtue of their causal powers grouped together into natural kinds or species. (Causal explanation, for Aristotle, is in terms of natural kinds and of the natural courses of development characteristic of the objects in each natural kind.) In each natural kind it is possible to distinguish (as Aristotle already saw) both natural or typical or standard instances, and also a penumbra of non-natural or non-typical or non-standard instances.

The families of natural kinds in each genus, for example the family of standard sound-types (phonemes) associated with a given language, manifest in each case the structure of a small finite space of readily distinguishable alternatives separated by gaps. Each typical instance of a substantial kind has its typical habits of motion, change and development, its typical overall style, its typical repertoire of the types of accidents which it is capable of acquiring and losing in typical sorts of circumstances.

Substances exist in space and their changes take place in time. Both space and time are unified in the sense that there ate no isolated points or regions of time, and also no isolated points or regions of space. Matter in contrast comes in separate chunks, all of which are such as to occupy finite regions of space for as long as they exist and all of which are (down to a certain level of granularity) continuous. Such substances are further capable of being divided, within limits, into pieces.

In addition to substances, qualities and processes, the common-sense world comprises also other varieties of non-substantial entity, for example liquids and gases (clouds, smoke, fire, etc.). Entities in these categories may be parcelled out in different ways. Liquids, for example, may be sortalized via quantities (pint of), shapes (drop or), disturbances (jet of), container (glass of), and so on. Common sense sees no problem in the fact that it can be useful to employ and to refer in this way to distinct parcellings of the same underlying materials.

This is indeed an aspect of the more general fact that the common-sense world is subject in all its parts and moments to the feature of continuity, a feature which goes hand in hand with the possibility of a drawing of internal and external boundaries. This holds first of all of substances (which take up space and endure through time, and therefore inherit from space and time a continuity of structure), of accidental qualities (which are spread out through space and are again such as to endure through time), and to processes (which unfold in time). It is true also of holes, shadows, diseases and other entities in non-traditional categories. "Continuity" here, however, should not be too hastily identified with continuity as this is standardly understood from the mathematical point of view. Rather, we are dealing with a qualitative notion of continuity, which is characterized by principles to the effect that a continuum can be divided into parts without initially specifiable limit, that the results of such division will share parts with the continuum before division, and that the division in question can in general be carried out along a plurality of different dimensions which are such as to cross-cut each other (Smith, in press). 


\section{Substances and substrates}

How, now, are we to understand the relation between this world of common-sense substances and that world of physical substrates which is the subject-matter of natural science? (For a detailed account of this problem, see Petitot (1994).) We note, first of all, that the Brentano-Husserl tradition of mereology includes also a topological component-a theory of continua and boundaries built up on a non-set-theoretic basis. Continua are seen by Brentano (1976) as wholes made up of two sorts of constituent: boundaries and extended parts. These two sorts of part are dependent upon each other in the sense that the existence of each boundary depends upon the existence of some larger extended whole which it is the boundary of, and the existence of each extended whole depends in turn upon the existence of its boundaries. Brentano (1976) himself used these ideas in providing an account of the structures of space and time, of perceptual qualities extended in space and time, and of three-dimensional bodies. Brentano's qualitative theory of the continuum can serve, now, as the basis for a general account of the ways in which the objects of the common-sense world are delineated within the larger realm of physical reality. Such objects are, as we have seen, parts or sub-regions of physical reality which are yet not recognized by or such as to be of interest to physical theory as such. As we stressed at the very start of this essay, they exist independently of human cognitive activities. On the other hand we can now recognize that they enjoy what might be called a delineation-dependent status, in the sense that it is our delineationsdelineations dependent in part upon the detailed make-up of our cognitive apparatus-which serve to set the corresponding sub-regions of physical reality into relief against the background of physical reality as a whole. Our cognitive activities thus do not bring common-sense objects into being from out of nowhere. They serve, rather, merely to delineate or discriminate the relevant underlying matter in light of manifest qualitative differences, and this matter is something which exists quite independently of our delineations.

Consider, for purposes of comparison, the way in which geographical boundary lines are drawn on a map. The State of Indiana is, we carl say, delineationdependent on certain geographical decisions made by Jefferson. Yet clearly Jefferson did not bring the underlying mass of territory into existence from out of nowhere. The objects of the common-sense world are in this respect analogous to geographical objects, and while it would take us too long to set out the details of this analogy here, the idea that such an analogy exists and that it is of central importance gains support from a wealth of results obtained already by cognitive scientists working in different fields on the phenomenon of systems of delineation of common-sense reality in vision research, natural-language semantics, studies of spatial reason, and elsewhere. $t$

\footnotetext{
t Thus we can point to the work of Talmy (1983) and others on the ways in which topological structures are reflected in the grammatical systems of natural languages, to the relevant work of Guarino (1992) and others in the field of representation of common-sense knowledge, to the work on spatial reasoning of Casati and Varzi (1994) and of Cohn and his associates (Randell \& Cohn, 1992), and to the work on mereology and topology as applied to the field of natural language semantics by Laure Vieu and her associates. (See for example, Aunague and Vieu, 1992.) Compare also the work on a general, universal ontology as a basis for an interlingua, e.g. on the part of Hovy and Nirenburg (1992).
} 


\section{Conclusion}

The program presented above might be represented, in simplified form, as follows: we take common sense as our guiding clue equally in ontology, psychology, semantics and metaphysics. We thus get a folk ontology (effectively mereology and topology), a folk psychology (especially of direct perception), a non-solipsistic and anti-representationalist semantics, a rationalist anthropology (innate ideas and structures derived from a priori principles governing the phenomenon of learning), and finally a theory of cultural universals. Clearly, not everyone will wish to buy this list as a whole. The paper is to this extent a position statement, embracing a most radical form of the common-sense realist view, and we are not expecting that all its readers will buy the whole package. To the extent that the resultant mereological and topological analyses are successful, however, these analyses may be of interest even to those who defend positions more moderate than that defended here. Thus for example they may be of interest to those who, while insisting that there is a significant diversity of natural ontologies, are nevertheless willing to admit that this diversity is constrained, for example by the fact that certain mereological and topological structures play a central organizing role in each. And they may be of interest to those who hold that an instrument with the power of set theory is needed for the purposes of formal semantics in order to cope with those natural language phenomena (for example counterfactuals, modal and intentional contexts) which seem less tractable from the non-representationalist point of view presented here. The mereotopological view of common-sense reality may even be of interest to the idealist, who sees world and objects as toto caelo a product of mind or language. For even the idealist must surely admit that such products would be structured in certain ways, and I venture to assert that at least part of the story of such structurings must involve mereotopological analyses of the sort here described.

\section{References}

Aurnague, M. \& Vieu, L. (1992). A three-level approach to the semantics of space. In The Semantics of Prepositions. From Mental Processing to Natural Language Processing. Berlin: Mouton/de Gruyter.

Brentano, F. (1967). Philosophische Untersuchungen zu Raum, Zeit und Kontinuum. (R. M. Chisholm \& S. Körner, Eds) Hamburg: Meiner. English translation, Philosophical Investigations on Space, Time and the Continuum, London: Croom Helm (1988).

BuNT, H. (1979). Ensembles and the formal semantic properties of mass terms. In F. J. Pelletier, Ed. Mass Terms. Some Philosophical Problems. Dordrecht: Reidel.

Campbell, K. (1988). Philosophy and common sense. Philosophy, 63, 161-174.

CASATI, R. \& VARZI, A. (1994). Holes and Other Superficialities. Cambridge, MA: MIT Press.

Chisholm, R. (1989). On Metaphysics. Minneapolis, MN: University of Minnesota Press.

Chisholm, R. M. (1984). Boundaries as dependent particulars. Grazer Philosophische Studien, 10, 87--95.

D'ANDrade, R. (1987). A folk model of the mind. In D. Holland \& N. Quinn, Eds. Cultural Models in Language and Thought. pp. 112-148 Cambridge: Cambridge University Press. 
Daniels, N. (1989). Thomas Reid's "Inquiry". The Geometry of Visibles and the Case for Realism. With a new Afterword, Stanford, CA. Stanford University Press.

Davis, E. (1990). Representations of Commonsense Knowledge. San Mateo, CA: Morgan Kaufmann.

Devitt, M. (1984). Realism and Truth. Oxford: Blackwell.

Douglas, J. D., (Ed.) (1973). Understanding Everyday Life. Toward the Reconstruction of Sociological Knowledge. London: Routledge and Kegan Paul.

DreTsKe, F. (1969). Seeing and Knowing. London: Routledge and Kegan Paul.

DREYFus, H. L. (1982). Husserl, Intentionality and Cognitive Science. Cambridge, MA: MIT Press.

Duncker, K. (1947). Phenomenology and epistemology of consciousness of objects. Philosophy and Phenomenological Research, 7, 1947.

Efron, R. (1966). What is perception?. Boston Studies in the Philosophy of Science, 4, 137-173.

Eschendach, C. \& HeydRICH, W. (1993). Classical mereology and restricted domains. In N. GuARINo \& R. Poll, Eds. International Workshop on Formal Ontology. Padua: Ladseb-CNR Internal Report 01/93.

Feyerabend, P. (1978) In defence of Aristotle: comments on the condition of content increase. In G. Radnitzky \& G. Andersson, Eds. Progress and Rationality. pp. 143-180. Dordrecht: Reidel.

Fine, K. (1995) Husserl's theory of part and whole. In B. Smith \& D. W. Smith, Eds. Cambridge Companion to Husserl. Cambridge: Cambridge University Press.

FoDOR, J. (1980). Methodological solipsism as a research strategy in cognitive psychology. Brain and Behavioral Sciences, 3, 63-73.

FodoR, J. \& PYLYSHYN, Z. (1981). How direct is visual perception? Some reflections on Gibson's "Ecological approach". Cognition, 9, 139-196.

Forguson, L. (1989). Common Sense. London: Routledge.

Gibson, J. J. (1966), The Senses Considered as Perceptual Systems. London: George Allen and Unwin.

GIBson, J. J. (1979). The Ecological Approach to Visual Perception. Boston, MA: HoughtonMiftin.

Guarino, N. (1992). Concepts, attributes and arbitrary relations. Some linguistic and ontological criteria for structuring knowledge bases. Data and Knowledge Engineering, 8, 249-261.

HAyes, P. J. (1985). The second naive physics manifesto. In J. R. Hoebs \& R. C. MoORE, Eds. Formal Theories of the Common-Sense World, pp. 1-36. Norwood: Ablex.

Hilbert, D. R. (1987) Color and Color Perception: A Study in Anthropocentric Realism, CSLI Lecture Note Series. Chicago, IL: University of Chicago Press.

Hobrs, J. R. \& Moore, R. C. Eds. (1985). Formal Theories of the Common-sense World. Norwood: Ablex.

HolthoON, F. van (1987). Common sense and natural law: from Thomas Aquinas to Thomas Reid. In F. van Holthoon \& D. R. Osson, Eds. Common Sense. pp. 99-114. Lanham/London: University Press of America.

Horton, R. (1982). Tradition and modernity revisited. In M. Hollts \& S. Lukes, Eds. Rationality and Relativism. pp. 201-260. Oxford; Blackwell.

Hovy, E. \& NirenderG, S. (1992). Approximating an interlingua in a principled way. Proceedings of the DARPA Speech and Natural Language Workshop, Hawthorne, NY, February, 1992.

Husserl, E. (1970a). Logical Investigations, 2 vols. (translated by J. N. FINDLAY) London: Routledge and Kegan Paul.

HUSSERL, E. (1970b). The Crisis of European Sciences and Transcendental Phenomenology. An Introduction to Phenomenological Philosophy. (translated by D. CARR) Evanston: Northwestern University Press.

Kelley, D. (1986). The Evidence of the Senses. London: Louisiana State University Press.

Lakoff, G. (1987). Women, Fire, and Dangerous Things. Chicago, IL: University of Chicago Press. 
Macnamara, J. (1986). A Border Dispute: The Place of Logic in Psychology. Cambridge, MA: MIT Press.

Menger, K. (1940). Topology without points. Rice Institute Pamphlets, 27, 80-107.

Moore, G. E. (1959). A defence of common sense. In G. E. MoOre, Ed. Philosophical Papers. pp. 32-59. London: George Allen and Unwin.

Mulligan, K. \& Smith, B. (1986). A relational theory of the act. Topoi, 5/2, 115-130.

NEISSER, U. (1987). From direct perception to conceptual structure. In U. NEISSER, Ed. Concepts and Conceptual Development. Ecological and Intellectual Factors in Categorization. pp. 11-24. Cambridge: Cambridge University Press.

Petitot, J. (1994). Phenomenology of perception, qualitative physics and sheaf mereology. In R. CASAti, B. Smith \& G. White, Eds. Philosophy and the Cognitive Sciences. Vienna: Hölder-Pichler-Tempsky.

Petrtot, J. \& Smith, B. (1990). New foundations for qualitative physics. In J. E. Tiles, G. T. McKee \& C. G. Dean, Eds. Evolving Knowledge in Natural Science and Artificial Intelligence. pp. 231-249. London: Pitman Publishing.

Petitot, J. \& Smith, B. (in press) Physics and the phenomenal world. In R. Poli \& P. M. Simons, Eds. Formal Ontology. Dordrecht: Kluwer.

Pitrenger, J. B. \& Runeson, S. (1990). Paolo Bozzi's studies of event-perception. ISEP [International Society for Event Perception], IV.

RANDEl, D. A. \& Cohn, A. G. (1992a). Modelling topological and metrical properties in physical processes. Principles of Knowledge Representation and Reasoning. Proceedings of the First International Conference, Toronto, Canada.

Randell, D. A. \& COHN, A. G. (1992b). Exploiting lattices in a theory of space and time. Computers and Mathematics with Applications, 23, 459-476.

Randell, D. A., Cui, Z. \& Cohn, A. G. (1992a). A spatial logic based on regions and connection. Principles of Knowledge Representation and Reasoning. Proceedings of the Third International Conference, Cambridge, MA., pp. 165-176.

Randell, D. A., Cul, Z. \& Cohn, A. G. (1992b). An interval logic for space based on "connection". In B. NeumanN, Ed. 10th European Conference on Artificial Intelligence. pp. 394-398. New York, NY: John Wiley and Sons.

Sellars, W. F. (1963). Philosophy and the scientific image of man. In Science, Perception and Reality. London; Routledge and Kegan Paul.

Simons, P. M. (1987). Parts. A Study in Ontology. Oxford: Clarendon Press.

Smith, B. Ed. (1982). Parts and Moments. Studies in Logic and Formal Ontology. Munich: Philosophia.

SMith, B. (1991). Relevance, relatedness and restricted set theory. In G. SCHURZ \& G. J. W. DORN, Eds. Advances in Scientific Philosophy. Essays in Honour of Paul Weingartner. pp. 45-56. Amsterdam: Rodopi.

Smith, B. (1992). Characteristica universalis. In K. Mulligan, Ed. Language, Truth and Ontology (Philosophical Studies Series). pp. 50-81. Dordrecht: Kluwer.

SMith, B. (1994). Review of Davis 1990. Machines and Mentality, 4, 245-249.

Smith, B. (1995). Common sense. In B. Smith \& D. W. SMITH, Eds. The Cambridge Companion to Husserl. New York, NY: Cambridge University Press.

SMith, B. (in press) On the coincidence of boundaries. In L. H. HAHN, Ed. The Philosophy of Roderick Chisholm (Library of Living Philosophers). La Salle: Open Court.

Smith, B. \& CASATI, R. (1994). Naive physics: an essay in philosophy. Philosophical Psychology, 7, 225-244.

TAlmy, L. (1983). How language structures space. In H. PICK \& L. Acredolo, Eds. Spatial Orientation: Theory, Research, and Application. pp. 225-282. New York, NY: Plenum Press.

Tном, R. (1988). Esquisse d'une sémiophysique. Paris: Interéditions. Englich translation by V. Meyer, Semio Physics: A Sketch. Aristotelian Physics and Catastrophe Theory. Redwood City, etc.: Addison-Wesley, 1990.

Wiggins, D. (1980). Sameness and Substance. Oxford: Blackwell.

Wiszecki, G. \& Stiles, W. S. (1982). Color Science. New York, NY: Wiley. 